\title{
VARIABLES ANTROPOMÉTRICAS, HÁBITOS Y DIETAS ALIMENTARIAS EN ADOLESCENTES Y JÓVENES: DIFERENCIAS EN FUNCIÓN DEL SEXO
}

\section{ANTHROPOMETRIC VARIABLES, EATING HABITS AND DIETS IN ADOLESCENTS AND YOUTH: SEX DIFFERENCES}

\author{
CARMEN MAGANTO ${ }^{1}$, MAITE GARAIGORDOBIL ${ }^{1}$ Y \\ LOREA KORTABARRIA ${ }^{1}$
}

Cómo referenciar este artículo/How to reference this article:

Maganto, C., Garaigordobil, M. y Kortabarria, L. (2016). Variables antropométricas, hábitos y dietas alimentarias en adolescentes y jóvenes: diferencias en función del sexo [Empathy in adolescence [Anthropometric variables, eating habits and diets in adolescents and youth: Sex differences]. Acción Psicológica, 13(2), 89-100. http://dx.doi.org/10.5944/ap.13.2.17817

\section{Resumen}

El estudio tuvo como objetivo analizar las diferencias entre sexos en variables antropométricas (reales, percibidas y deseadas), en hábitos alimentarios, y en el uso de dietas alimentarias. Los participantes fueron 1.075 adolescentes y jóvenes de 14 a 25 años $(49.9 \%$ varones, $50.1 \%$ mujeres). Con un diseño descriptivo y comparativo, se administraron tres instrumentos de evaluación. Los resultados confirman muchas diferencias significativas entre sexos. En variables antropométricas las chicas se perciben más obesas de lo que están y desean estar más delgadas; los chicos se perciben igual o
\end{abstract}

más delgados de lo que están y desean tener un volumen corporal superior. Los chicos desean tener un Índice de Masa Corporal (IMC) superior y las chicas inferior. Las chicas obtienen puntuaciones significativamente superiores en hábitos alimentarios, aunque los chicos perciben que tienen una alimentación más equilibrada. Las chicas han realizado más dietas y creen necesitarlas más. Las razones para engordar en los chicos son biológicas $\mathrm{y}$ en las chicas hábitos alimentarios inadecuados. Las chicas realizan más dietas tanto saludables como no recomendables. Las razones para comenzar una dieta son en las chicas la imagen corporal y en los chicos ser aceptado por los iguales. El abandono de las dietas los chicos lo atribuyen a la dieta y las chicas a

Agradecimientos: Estudio financiado por el Dpto. de Educación, Política Lingüística y Cultura del Gobierno Vasco (IT638-13), y por la Universidad del País Vasco UPV/EHU (UFI PSIXXI 11/04).

Correspondencia: Carmen Maganto. Facultad de Psicología. Universidad del País Vasco (UPV/EHU).

Email: carmen.maganto@ehu.eus

${ }^{1}$ Universidad del País Vasco, España.

Recibido: 8 de abril de 2016.

Aceptado: 5 de julio de 2016 . 
sí mismas. El estudio aporta datos relevantes para el diseño de programas preventivos y/o de tratamiento con adolescentes/jóvenes con problemas alimentarios, bien por alteraciones de la imagen corporal, hábitos alimentarios inadecuados y/o por el uso indebido de dietas alimentarias.

Palabras clave: variables antropométricas; hábitos alimentarios; dietas; sexo; adolescentes y jóvenes.

\begin{abstract}
The study aimed to analyze sex differences in anthropometric variables (real, perceived, and desired), eating habits, and the use of diets. Participants were 1,075 adolescents and youth aged 14 to 25 (49.9\% males, $50.1 \%$ females). Using a descriptive and comparative design, three assessment instruments were administered. The results confirm many significant sex differences. In anthropometric variables, girls perceive themselves as more obese than they are and they want to be thinner; boys perceive themselves as thin as or thinner than they are and they want to have greater body volume. Boys want to have a higher Body Mass Index (BMI), and girls want a lower one. Girls score significantly higher in eating habits, although boys perceive their diet as more balanced. The girls have followed more diets and believe they need them more. In boys, the reasons for weight gain are biological and in girls, due to improper eating habits. Girls follow more diets, both healthy and not recommended. In girls, the reason for starting a diet is body image and in boys, peer acceptance. Boys attribute quitting a diet to the diet itself, and girls attribute it to themselves. The study provides relevant data for the design of prevention and/or treatment programs targeting adolescents/youth with eating problems, either due to alterations in body image, unsuitable eating habits, and/or misuse of diets.
\end{abstract}

Keywords: anthropometric variables; eating habits; diet; sex; adolescents and youth.

\section{Introducción}

Las variables antropométricas de peso, altura e IMC han sido objeto de estudio en relación con Hábitos Alimentarios (HA) y Dietas Alimentarias (DA). El deseo de poseer un cuerpo más delgado en las mujeres y más musculoso en los varones da lugar a problemas de percepción del propio cuerpo (Sehm y Warschburger, 2015). Las adolescentes tienden a percibirse más obesas de lo que están (peso percibido distorsionado) y a desear un cuerpo más estilizado (peso deseado y peso ideal de mayor delgadez), teniendo estas variables (peso percibido, peso deseado y peso ideal) mayor incidencia en las alteraciones alimentarias que el propio peso objetivo o peso real (Calzo et al., 2015; Maganto y Cruz, 2008).

Los estudios evidencian que cuando el IMC ideal está muy por debajo del IMC real, unido a baja autoestima, se convierte en una razón para desear bajar peso de forma rápida y excesiva, y como consecuencia aparecen alteraciones alimentarias (Berner, Shaw, Wirr y Lowe, 2013). Para Loth, MacLehose, Bucchianeri, Crow y NeumarkSztainer (2014) un bajo o alto IMC está asociado a la satisfacción corporal, pero confirman que es la percepción del tamaño corporal la que explica los comportamientos dirigidos a la pérdida de peso, utilizando el control del peso para mostrar su autocontrol y para sentirse competentes, queriendo en todo momento tener un IMC ideal por debajo del que tienen. Esta diferencia entre peso percibido, real y deseado es especialmente significativa en la adolescencia, ya que las jóvenes tienden a disminuir la diferencia entre estos valores. Asuero, Avargues, Martín y Borda (2012) en su investigación con una muestra de 146 chicas mayores de 16 años y con Trastornos de Conducta Alimentaria (TCA) obtuvieron resultados que mostraban que las chicas se percibían con un peso superior al que tenían.

En cuanto a los HA, al ser la adolescencia una etapa de cambios y poca estabilidad psicosocial y emocional, su alimentación depende más de la "moda" que de decisiones saludables, adoptando estilos de vida poco o nada saludables. Este cambio está relacionado con la modificación del estilo de vida en general y la organización familiar en particular (Aranceta, 2013). Actualmente, cada persona consume bastantes más calorías de las que 
necesita y más de las que consumía años atrás, los productos frescos están dejando paso a los alimentos precocinados y las bebidas con gas y las bebidas alcohólicas están sustituyendo a las bebidas saludables. Se ha demostrado que especialmente los chicos abusan más de la carne e ingieren menos verdura (Borobio, 2012). Rodríguez et al. (2013) concluyen que los estudiantes universitarios no desayunan ni ingieren alimentos entre las comidas centrales, ayunando durante largas horas durante el día y prefiriendo comer comida rápida rica en grasas, mostrando una adherencia media-baja a la dieta mediterránea. Esta tendencia se está produciendo tanto en países europeos como fuera de Europa.

En España, uno de los estudios más rigurosos (SerraMajem y Aranceta, 2012) muestra que entre 14 a 25 años se tiene un consumo moderado de leche, un alto consumo de carne y un bajo consumo de pescado, fruta y verdura. La Encuesta Nacional de Ingesta Dietética (2014), destaca la preferencia que tienen adolescentes y jóvenes por la pasta y el arroz frente a frutas y verduras, no siguiendo las recomendaciones nutricionales ni la mitad de jóvenes (solo $45.4 \%$ consume fruta diariamente y el $15.9 \%$ verduras). La carne es uno de los alimentos preferidos mientras que el pescado es la proteína animal que menos les gusta, consumiendo en exceso aceites vegetales, carne, embutidos, dulces, snacks, bollería y grasas, esto es, alimentos ricos en lípidos y colesterol.

Estos HA inadecuados se acompañan de DA inadecuadas, especialmente en mujeres adolescentes. El deseo de bajar peso en poco tiempo conlleva realizar dietas que en nada cumplen los requisitos de una nutrición saludable. Saucedo y Unikel (2010) informan que estas conductas de riesgo nutricional se daban principalmente en mujeres con sobrepeso $(13.3 \%)$ y en varones con obesidad (15\%), concluyendo que no son exclusivas del sexo femenino.

Hay estudios que afirman (Eisenberg y NeumarkSteiner, 2013; Goldschmidt, Wall, Loth, Le Grange y Neumark-Sztainer, 2012) que la obsesión por ponerse a dieta y realizar restricciones alimentarias puede ser el inicio de los TCA (Racine, Burt, Iacono, McGue y Klump, 2011), puesto que cuanto más restrictiva es la dieta, mayor es el riesgo de desarrollar un TCA (Berner et al., 2013; Liechty y Lee, 2013) o aumentar el riesgo de problemas de salud, es decir, aumento de peso (NeumarkSztainer, Wall, Haines, Story y Eisenberg, 2007), atracones, bulimia, depresión y baja autoestima en adolescentes (Racine et al., 2011).

Neumark-Sztainer et al. (2007) encontraron que el $55.4 \%$ y $24.8 \%$ de chicas y chicos respectivamente, informaron hacer dieta, poniéndose en una posición de mayor vulnerabilidad a la adopción de hábitos alimenticios inadecuados. El ayuno estuvo presente en el $23 \%$ de los adolescentes con puntuaciones altas en restricción de nutrientes básicos. Se ha comprobado que la "moda" en las dietas atrae a las personas más jóvenes practicando cualquier tipo de dieta proveniente de los pares, revistas del corazón, divulgación publicitaria o remedios caseros, sin considerar necesaria la consulta dietética profesional. Revisadas las dietas que más comúnmente llevan a cabo adolescentes y jóvenes encontramos una lista recogida en el Cuestionario de Dietas Alimentarias (Kortabarria, Maganto y Peris, 2013).

De entre todas las dietas que existen en nuestro contexto, la dieta más saludable, es la mediterránea, que no solo garantiza un aporte calórico y de nutrientes en cantidades suficientes y proporciones adecuadas, sino que contribuye a la prevención de enfermedades cardiovasculares, diabetes, cáncer, enfermedades degenerativas $\mathrm{y}$, en general, a una mayor esperanza de vida (Durá y Castroviejo, 2011). También se ha incluido la dieta vegetariana, considerada una dieta saludable al comprobar que está relacionada con buena salud, buenos hábitos alimentarios y con un peso adecuado (Timbo, Hormes y Chubski, 2012).

\section{Objetivos e hipótesis}

Teniendo en cuenta los antecedentes citados, el objetivo de este estudio es analizar en adolescentes y jóvenes las diferencias entre sexos en tres tipos de variables: (1) variables antropométricas reales, percibidas y deseadas (peso, altura e IMC), (2) hábitos alimentarios (ítems de desayuno, primer plato, segundo plato, postre y bebidas), y (3) dietas alimentarias (razones para engordar, 
seguimiento de dietas, razones para hacer dietas y razones para abandonar las dietas). Con estos objetivos se plantean tres hipótesis: (1) El IMC percibido en relación al IMC real será superior en las chicas que en los chicos, mientras que el IMC deseado será superior en los chicos que en las chicas; (2) Los hábitos alimentarios serán más saludables en los chicos que en las chicas; y (3) En relación a las dietas, se espera encontrar que las chicas realicen más dietas, engorden por hábitos alimentarios inadecuados, inicien dietas por problemas de imagen corporal y las abandonen por falta de voluntad, y que los chicos perciban que se engorda por motivos biológicos, hagan dietas para conseguir aceptación por parte de los demás, y las abandonen por las características de la propia dieta.

\section{Método}

\section{Participantes}

Los participantes fueron 1.075 de los cuales 536 son varones $(49.9 \%)$ y 539 mujeres $(50.1 \%)$. En función de la edad, $507(47.2 \%)$ son adolescentes de 14 a 18 años y $568(52.8 \%)$ jóvenes de 19 a 25 . Se trata de una muestra comunitaria representativa de estudiantes de ESO, Bachiller y Universidad del País Vasco. La muestra fue seleccionada mediante una técnica de muestreo aleatorio simple a partir de la lista de centros educativos de Educación Secundaria y universitarios del País Vasco. Según la última encuesta del Instituto Vasco de Estadística (eustat.es), la población de la ESO y Bachiller en el País Vasco es 101.757, y los que cursan estudios universitarios 64.127. Utilizando un nivel de confianza de .95, con un error muestral de .05 , para una varianza poblacional de .50 , la muestra representativa sería de 766 participantes.

\section{Instrumentos de evaluación}

Variables antropométricas reales, percibidas y deseadas: Los participantes fueron pesados y medidos utilizando básculas digitales, a fin de obtener el peso, altura y el IMC real (peso/estatura ${ }^{2}$ ). El peso, altura e IMC percibido se obtuvieron a través de tres preguntas:
En una escala del 1 (muy delgado) a 10 (muy gordo) ¿Cómo percibes tu peso? ¿Cómo percibes tu altura? (1 muy baja a 10 muy alta) ¿Cómo percibes tu IMC? (1 muy delgado a 10 muy grueso). Además, se añadió la siguiente información y preguntas: El IMC representa el volumen total de tu cuerpo en proporción a lo que mides. Un IMC muy bajo (1) corresponde a personas muy delgadas y un IMC muy alto (10) corresponde a personas con mucho volumen corporal, que pesan mucho en proporción con lo que miden. Para obtener el peso y la altura deseados, se añadieron dos preguntas: ¿Cuánto te gustaría pesar? ¿Cuánto te gustaría medir? Para obtener el IMC deseado se llevó a cabo la ecuación de peso deseado dividido por la altura deseada al cuadrado.

Cuestionario de alimentación (trabajoyalimentación.consumer.es). Tras la revisión realizada sobre instrumentos de HA se seleccionó el autoinforme diseñado y avalado por el Departamento de Investigación Alimentaria de la Fundación Eroski. Este cuestionario es un autoinforme que tiene en cuenta los componentes básicos de los alimentos: lácteos, cereales, proteínas y vitaminas, carbohidratos y grasas, agrupados en desayuno, primer y segundo plato y postre, incluyendo las bebidas. Consta de 17 ítems valorados según una escala tipo Likert del 1 al 3, donde 1 es siempre el valor más negativo y 3 el más positivo. A mayor puntuación mejores hábitos alimentarios. En este estudio el Alpha de Cronbach fue de .61, coeficiente que se puede considerar adecuado teniendo en cuenta que nadie realiza una dieta absolutamente equilibrada y que los ítems exploran valores muy diversos entre sí.

Dietas alimentarias: En ausencia de instrumentos estandarizados sobre dietas alimentarias, se ha diseñado un cuestionario "ad hoc" para la investigación. Se partió del concepto de cuestionarios en el sentido propio del término (Morales, 2011), es decir, una lista de preguntas que se proponen con fines diversos, en los que las respuestas se analizan de manera independiente, por lo que las propiedades psicométricas no tienen cabida. Se inicia con un sondeo sobre dietas (tu alimentación es equilibrada, has hecho dieta, necesitas hacer dieta, alguien de tu familia ha hecho dieta). Le siguen cuatro aspectos generales: (1) Razones para engordar, que contempla dos categorías: razones biológicas (genética, tiroides, ingesta 
de medicamentos, personas propensas, nervios) y razones de hábitos alimentarios inadecuados (comer excesivamente, comer inadecuadamente, no hacer ejercicio y dejar el tabaco); (2) Seguimiento de dietas en el que se analizan: dietas saludables (mediterránea y vegetariana), dietas con productos farmacéuticos (barritas saciantes, sopas quemagrasas; productos eliminagrasas) y dietas poco saludables (Montignac, Clínica Mayo, de limón y pomelo, y toma la mitad); (3) En cuanto a las razones para iniciar una dieta se analizan la salud (sobrepeso, prescripción médica, obligan los padres), razones de imagen corporal (rechazo del cuerpo, vestir ropa actual, seguir modelo de belleza) y la aceptación de iguales (aceptación de los demás; gustar a la pareja, los amigos lo hacen), y (4) En las razones para abandonar la dieta se analizan razones atribucionales a la propia dieta (dan hambre, ocasionan tristeza y depresión, crean ansiedad, enferman, no adelgazan lo que prometen) y razones personales (aburrimiento, falta de voluntad, era mal momento, meta poca realista).

\section{Procedimiento}

Para el desarrollo del estudio se llevaron a cabo las siguientes fases: (1) Se estableció contacto con los directores de los centros educativos ofreciendo información del estudio e invitándoles a colaborar; (2) Tras la aceptación, se solicitó consentimiento informado a la di- rección, a padres y participantes; y (3) Se administraron los instrumentos de evaluación de forma colectiva, voluntaria y anónima en el aula. El estudio cumplió los valores éticos requeridos en la investigación con seres humanos, respetando los principios fundamentales incluidos en la Declaración Helsinki y en las normativas vigentes.

\section{Análisis de datos}

Para analizar las diferencias entre sexos en las variables antropométricas, hábitos y dietas alimentarias se llevaron a cabo análisis descriptivos (medias y desviaciones típicas) y de comparación de medias (T-test), calculando el tamaño del efecto ( $d$ de Cohen). En las variables categóricas del cuestionario de dietas alimentarias se obtuvo la Chi cuadrado de Pearson.

\section{Resultados}

\section{Medidas antropométricas: diferencias entre sexos}

Las diferencias entre sexos en las variables antropométricas (peso, altura, IMC: reales, percibidos y deseados) se presentan en la Tabla 1.

Tabla 1

Medias, desviaciones típicas y resultados T-test en peso, altura e IMC real, percibido y deseado en ambos sexos

\begin{tabular}{|c|c|c|c|c|c|c|c|c|c|}
\hline & \multicolumn{2}{|c|}{$\begin{array}{c}\text { Total } \\
(N=1.075)\end{array}$} & \multicolumn{2}{|c|}{$\begin{array}{l}\text { Chicos } \\
(n=536)\end{array}$} & \multicolumn{2}{|c|}{$\begin{array}{l}\text { Chicas } \\
(n=539)\end{array}$} & \multirow[t]{2}{*}{$t$} & \multirow[t]{2}{*}{$p$} & \multirow[t]{2}{*}{$d$} \\
\hline & $M$ & $D T$ & $M$ & $D T$ & $M$ & $D T$ & & & \\
\hline Peso real & 62.62 & 10.65 & 69.61 & 10.15 & 59.31 & 9.61 & 16.29 & .001 & 1.04 \\
\hline Peso percibido & 4.95 & 1.39 & 4.62 & 1.16 & 5.14 & 1.52 & 5.16 & .001 & -0.38 \\
\hline Peso deseado & 60.18 & 9.61 & 70.25 & 8.04 & 55.01 & 6.13 & 33.93 & .001 & 2.13 \\
\hline Altura real & 1.69 & 0.08 & 1.76 & 0.06 & 1.64 & 0.06 & 29.04 & .001 & 2.00 \\
\hline Altura percibida & 5.53 & 1.97 & 5.93 & 1.66 & 5.14 & 2.08 & 5.72 & .001 & 0.41 \\
\hline Altura deseada & 1.72 & 0.08 & 1.80 & 0.05 & 1.67 & 0.04 & 40.11 & .001 & 2.87 \\
\hline IMC real & 21.85 & 3.07 & 22.23 & 2.83 & 21.85 & 3.09 & 1.96 & .050 & 0.12 \\
\hline IMC percibido & 4.75 & 0.90 & 4.50 & 0.82 & 4.92 & 0.92 & 7.79 & .001 & -0.48 \\
\hline IMC deseado & 20.01 & 2.09 & 21.47 & 2.02 & 19.49 & 1.90 & 15.99 & .001 & 1.00 \\
\hline
\end{tabular}


Tabla 2

Medias, desviaciones típicas y resultados T-test en hábitos alimentarios en ambos sexos

\begin{tabular}{|c|c|c|c|c|c|c|c|c|c|}
\hline & \multicolumn{2}{|c|}{$\begin{array}{c}\text { Total } \\
(N=1075)\end{array}$} & \multicolumn{2}{|c|}{$\begin{array}{c}\text { Chicos } \\
(n=536)\end{array}$} & \multicolumn{2}{|c|}{$\begin{array}{c}\text { Chicas } \\
(n=539)\end{array}$} & \multirow[t]{2}{*}{$t$} & \multirow[t]{2}{*}{$p$} & \multirow[t]{2}{*}{$d$} \\
\hline & $M$ & $D T$ & $M$ & $D T$ & $M$ & $D T$ & & & \\
\hline Desayuno y postre recomendable & 9.51 & 1.53 & 9.53 & 1.62 & 9.44 & 1.46 & 0.93 & .348 & 0.05 \\
\hline Primeros y segundos platos recomendables & 11.59 & 1.57 & 11.43 & 1.47 & 11.75 & 1.64. & 3.34 & .001 & -0.20 \\
\hline Alimentos poco recomendables & 6.87 & 1.46 & 7.07 & 1.42 & 6.69 & 1.47 & 4.24 & .001 & 0.26 \\
\hline Bebidas poco recomendables & 4.17 & 1.02 & 4.47 & 1.12 & 3.93 & 0.86 & 8.81 & .001 & 0.54 \\
\hline
\end{tabular}

En la Tabla 1, las diferencias estadísticamente significativas entre chicos y chicas muestran que las chicas pesan menos, se perciben más gruesas de lo que están $\mathrm{y}$, por consiguiente, aún les gustaría pesar menos. Los chicos además de medir más que las chicas, también desearían ser más altos que lo que ellos son y más altos de lo que a las chicas les gustaría ser. Respecto al IMC deseado, también a los chicos les gustaría que fuera superior de lo que es, mientras que a las chicas les gustaría que fuera menor de lo que es, pues perciben su IMC superior al que en realidad tienen y superior a como los chicos perciben su IMC.

\section{Hábitos alimentarios: Diferencias entre sexos}

Con la finalidad de analizar las diferencias en función del sexo en el cuestionario de hábitos alimentarios, se agruparon los ítems en 4 categorías en función del criterio hábitos recomendables versus no recomendables, y se realizaron análisis de T-test cuyos resultados se exponen en la Tabla 2.

Como se observa en la Tabla 2, en la ingesta adecuada del desayuno y postre, las diferencias no son estadísticamente significativas, pero sí lo son en los primeros y segundos platos, ya que las chicas obtienen puntuaciones superiores a los chicos, realizando ellas

\section{Tabla 3.}

Frecuencias, porcentajes y resultados de $x^{2}$ en variables relacionadas con alimentación y dieta en ambos sexos

\begin{tabular}{|c|c|c|c|c|c|c|}
\hline & \multicolumn{2}{|c|}{$\begin{array}{c}\text { Chicos } \\
(n=536)\end{array}$} & \multicolumn{2}{|c|}{$\begin{array}{c}\text { Chicas } \\
(n=539)\end{array}$} & \multirow[t]{2}{*}{$\chi^{2}(1)$} & \multirow[t]{2}{*}{$p$} \\
\hline & $F$ & $\%$ & $F$ & $\%$ & & \\
\hline Alimentación equilibrada & 373 & 85.4 & 389 & 79.6 & 5.33 & .025 \\
\hline Seguimiento de dietas & 84 & 15.8 & 243 & 45.3 & 109.25 & .001 \\
\hline Necesidad hacer dieta & 64 & 12.1 & 246 & 46.1 & 148.18 & .001 \\
\hline Dietas en familiares & 354 & 67.4 & 444 & 83.3 & 35.95 & .001 \\
\hline
\end{tabular}

menor ingesta de alimentos y bebidas poco recomendables, pudiendo afirmar que los chicos tienen hábitos alimentarios menos recomendables que las chicas. No se encontraron diferencias entre sexos en la ingesta de lácteos.

\section{Dietas alimentarias: Diferencias entre sexos}

Las diferencias entre sexos en el cuestionario de dietas alimentarias se visualizan en las Tablas 3 y 4 . El cuestionario se inicia con un sondeo sobre alimentación y dietas (percepción del equilibrio de su alimentación, uso de dietas, percepción de necesidad de hacer dieta, realización de dietas en la familia) cuyos resultados se presentan en la Tabla 3. Posteriormente el cuestionario estudia cuatro aspectos en relación a las dietas (razones por las que se engorda, seguimiento de dietas, razones por las que se inicia una dieta y razones por las que se abandonan las dietas) cuyos resultados se presentan en la Tabla 4.

Los chicos perciben significativamente su alimentación más equilibrada que las chicas (ver Tabla 3), a pesar de que se ha podido comprobar que son estas últimas las que obtienen puntuaciones superiores en los hábitos alimentarios (ver Tabla 2). Además, donde las 
diferencias estadísticamente significativas son más evidentes es en las variables he hecho dieta o necesito hacer dieta, ya que, mientras casi la mitad de las mujeres han hecho dieta o dicen que necesitan hacerla, sólo un $15.8 \%$ y un $12.1 \%$ de los chicos contestan afirmativamente en estas variables. Además, las mujeres significa- tivamente informan tener más familiares que han hecho dieta.

Como se puede observar en la Tabla 4, las diferencias estadísticamente significativas en razones para engordar son el mal funcionamiento de la tiroides con puntuacio-

Tabla 4

Medias, desviaciones típicas y resultados T-test en el cuestionario de dietas alimentarias en ambos sexos

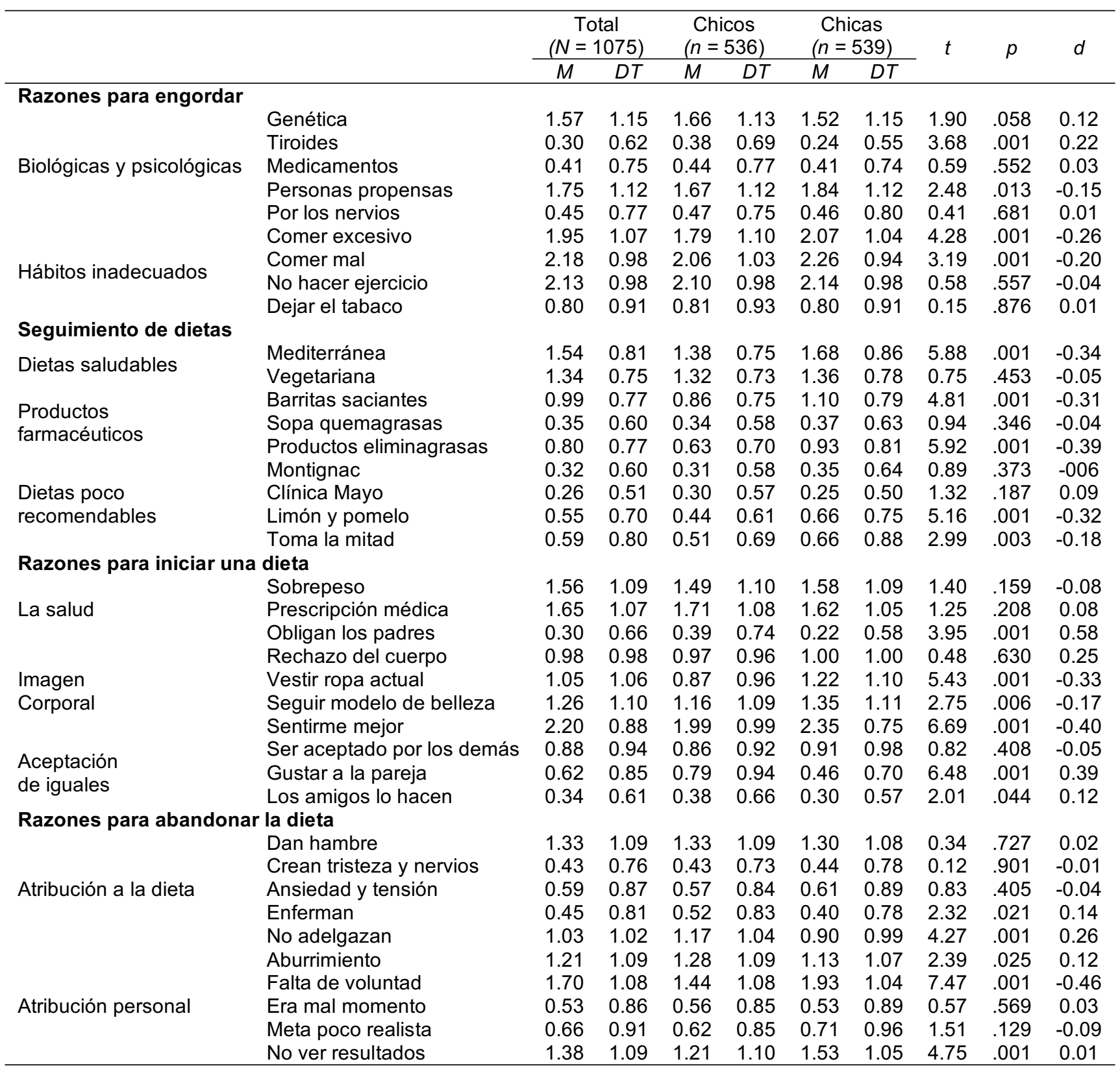


nes superiores en los chicos, y la propensión a engordar con puntuaciones superiores en las chicas. En cuanto a los hábitos alimentarios inadecuados las diferencias entre chicos y chicas, a favor de éstas últimas, son comer excesivamente y comer mal, cosas que engordan. En ambos casos las diferencias son estadísticamente significativas a favor de las chicas. No hay diferencias entre sexos en las razones atribuidas a la genética, ingesta de medicamentos, por nervios, no hacer ejercicio físico y dejar el tabaco.

En el seguimiento de dietas, las chicas han realizado mayor número de dietas que los chicos, obteniendo puntuaciones significativamente superiores en: dieta mediterránea, barritas saciantes, productos elimina-grasas, dieta de limón y pomelo y dieta de toma la mitad. No se han hallado diferencias significativas en la dieta vegetariana, sopas eliminagrasas, dieta Montignac y Clínica Mayo.

Respecto a las razones para comenzar una dieta, las chicas significativamente aportan solo razones de imagen corporal: ponerse ropa actual, seguir el modelo de belleza y sentirse mejor conmigo misma. En los chicos las razones son significativamente superiores en sentirse obligado por los padres, gustar a la pareja y que los amigos lo hacen. No hay diferencias entre sexos en las siguientes razones: sobrepeso, prescripción médica, rechazo del cuerpo y ser aceptado por los demás.

Dentro de las razones para abandonar la dieta, los chicos obtienen puntuaciones significativamente superiores a las chicas en la razón de que se puede enfermar o que no adelgazan lo prometido. Dentro de la razón de atribución personal, los chicos obtienen puntuaciones significativamente superiores a las chicas en que son aburridas, mientras que las chicas obtienen puntuaciones superiores en la razón de falta de voluntad y no ver los resultados rápidos. No se encontraron diferencias entre sexos en las razones siguientes: dan hambre, crean tristeza y ansiedad, era mal momento, la meta era poco realista.

\section{Discusión}

El estudio tuvo como objetivo analizar las diferencias en función del sexo en variables antropométricas, hábitos alimentarios y uso de dietas. La hipótesis 1 proponía que las chicas se percibirían significativamente más gruesas que los chicos y en el peso deseado las chicas desearían pesar menos de lo que pesaban, mientras que los chicos desearían aumentar de peso.

Los resultados confirman que los chicos obtienen puntuaciones estadísticamente superiores a las chicas en peso y altura. Sin embargo, en peso percibido y en IMC, ellas se perciben más obesas que ellos, deseando pesar menos de lo que pesan, tal y como se puede comprobar al comparar el peso deseado con el peso real, mientras que a los chicos les gustaría aumentar de peso (Calzo et al., 2015; Maganto y Cruz, 2008; Sehm y Warschburger, 2015). Los resultados permiten confirmar la hipótesis 1 . El hecho de percibirse las mujeres con un tamaño corporal superior al real y un peso real superior al ideal promueve insatisfacción corporal, siendo a su vez una de las razones que promueve adelgazar. Esta diferencia entre peso percibido y peso deseado se convierte en factor de riesgo de dietas repetidas e inadecuadas, como se observa en otros estudios (Berner et al., 2013). Cuanto mayor es la distancia entre IMC real e IMC deseado mayor insatisfacción corporal y mayor riesgo de padecer un TCA (Loth et al., 2014). Esta diferencia entre peso percibido, real y deseado es especialmente significativa en la adolescencia, ya que tiende a disminuir la diferencia entre estos valores a cualquier precio. Analizando los resultados de los chicos vemos, por el contrario, que les gustaría pesar más y ser más altos, lo que supondría ser más atractivos al ganar en masa muscular, asociada a fuerza, actitud activa, seguridad y poder.

La segunda hipótesis proponía que los chicos obtendrían puntuaciones significativamente superiores a las chicas en HA adecuados. Los resultados muestran una alimentación significativamente más saludable en chicas que en chicos al ingerir de manera más adecuada los primeros y segundos platos y abusar menos de alimentos poco recomendados, como fritos, dulces y comida precocinada, e ingerir menos bebidas poco recomendables (alcohólicas, energetizantes, gaseadas). De- 
bido a ello, no se confirma la hipótesis 2 , ya que los chicos tienen una alimentación menos adecuada que las chicas.

Estos resultados pueden ser explicados por la asociación que las mujeres realizan entre delgadez y belleza al interiorizar el ideal estético social de delgadez y concuerdan con estudios precedentes (Sehm y Warschburger, 2015), considerando este modelo algo natural, perfecto y sano, promoviendo el deseo de estar delgadas y seguir dietas para reducir peso. De este modo, las chicas consumen menos grasas e ingieren alimentos más saludables, como las frutas y verduras, mientras que los chicos consumen menos frutas y verduras y más carne y bebidas gaseosas, por lo que los HA y DA de los chicos son menos equilibrados y saludables (Borobio, 2012). Saucedo y Unikel (2010) obtuvieron resultados divergentes, manifestando que las chicas, bien por su preocupación por el aspecto físico o bien por el miedo a engordar, son las que tenían HA de mayor riesgo de TCA y DA de peor calidad. Éstas eliminan alimentos con alto contenido de lípidos, ayunan de días completos y restringen tanto alimentos poco recomendados como saludables, exponiéndose al riesgo que padecer un TCA (Encuesta de Salud, 2013; Rodríguez et al., 2013).

Aunque estos resultados parecen contradictorios, no lo son si se tienen en cuenta que el deseo de delgadez lleva consigo seleccionar alimentos más saludables (frutas y verduras) y restringir los que más engordan (grasas, alcohol, dulces, fritos y bebidas carbonatadas), y en este sentido las mujeres se alimentan mejor; pero, cuando la obsesión por la delgadez es elevada, la alimentación termina en dietas aberrantes, restricciones alimentarias extremas, y errores nutricionales de riesgo, confirmando lo obtenido en otros estudios (Saucedo y Unikel, 2010).

En cuanto a las dietas alimentarias, la hipótesis tercera planteaba que las chicas realizarían más tipos de dietas, creían necesario hacer dietas, las hacían por insatisfacción corporal y las abandonarían por falta de voluntad. Efectivamente, las chicas obtienen puntuaciones significativamente superiores a los chicos ya que realizan más tipos de dietas: mediterránea, barritas saciantes, productos eliminagrasas, limón y pomelo y toma la mitad. También mayor porcentaje de chicas hacen dieta, aduciendo razones de imagen corporal (ponerse ropa actual, seguir el modelo de belleza o sentirse mejor consigo mismas) y las abandonan por falta de fuerza de voluntad y no ver resultados rápidos. Estos resultados confirman la hipótesis 3 y van en consonancia con los de Eisenberg y Neumark-Steiner (2013), quienes encontraron que las chicas siguen más tipos de dietas que los chicos, tanto saludables (Durá y Castroviejo, 2011), como no saludables, siendo la imagen corporal una razón de peso para ello (Kortabarría et al., 2013). Apenas existen estudios que analicen las variables estudiadas en la presente investigación, es decir, razones de iniciar una dieta, tipo de dietas y motivos para abandonarlas, pero los que existen confirman que las mujeres frente a los varones y los adolescentes frente a los jóvenes realizan dietas menos saludables y no las hacen por motivos de salud, existiendo mayor riesgo de TCA (Berner et al., 2013; Goldschmidt et al., 2012; Liechty y Lee, 2013; Loth et al., 2014; Racine et al., 2011).

Las principales aportaciones del estudio se centran en la desigualdad de los HA en varones y mujeres, teniendo que tener cuidado si, siendo aparentemente mejores los de mujeres, son en definitiva hábitos excesivamente restrictivos en grasas y otros nutrientes considerados que engordan, pero absolutamente necesarios en los HA saludables. Otra aportación significativa ha sido clarificar la importancia que tiene la distancia entre peso real, percibido y deseado, especialmente para las mujeres, como factor de riesgo de la distorsión e insatisfacción corporal y las consecuencias que conllevan para el inicio de HA y DA inadecuadas, aberrantes y excesivamente restrictivas. La mayor aportación ha consistido en el diseño de un instrumento de evaluación de DA para analizar distintos aspectos relacionados con las dietas alimentarias en adolescentes y jóvenes, teniendo en cuenta la ausencia de dichos instrumentos y la importancia de los mismos en los TCA.

Sin embargo, hay dos limitaciones a señalar. Una, que el estudio está realizado con autoinformes, por lo que el sesgo de deseabilidad social puede en parte alterar los resultados. Otra limitación deriva de la ausencia de instrumentos de evaluación sobre HA y DA, por lo que la verificación de la hipótesis queda reducida. 
Sin embargo, los resultados de este estudio en relación a la atribución que hacen sobre razones para engordar, iniciar una dieta y abandonarla abren un amplio campo de investigación, tanto desde el punto de vista de la evaluación-intervención, en la que habría que consolidar la construcción de instrumentos con características psicométricas adecuadas, ya que proveería de importantes datos en el ámbito de la patología alimentaria y en el de la prevención-intervención en los TCA.

\section{Referencias}

Aranceta, J. (2013). Nutrición Comunitaria. Barcelona, España: Toray Masson.

Asuero, R., Avargues, M. L., Martín, B. y Borda, M. (2012). Preocupación por la apariencia física y alteraciones emocionales en mujeres con trastornos alimentarios con autoestima baja. Escritos de Psicología, 5(2), 39-45. http://dx.doi.org/10.5231/psy.writ.2012.1007

Berner, L. A., Shaw, J. A., Witt, A. A. y Lowe, M. R. (2013). The relation of weight suppression and body mass index to symptomatology and treatment response in anorexia nervosa. Journal of Abnormal Psychology, 122(3), 694-708. http://dx.doi.org/:10.1037/a0033930

Borobio, A. (2012). Dieta y alimentos. Estudio sobre el conocimiento de los hábitos alimenticios y la eficacia de la educación nutricional en $3^{\circ}$ de ESO. Universidad Internacional de La Rioja. Recuperado de http://reunir.unir.net/handle/123456789/569

Calzo, J. P., Masyn, K., Corliss, H., Scherer, E. A., Field, A. E. y Austin, N. (2015). Patterns of body image concerns and disordered weight- and shape-related behaviors in heterosexual and sexual minority adolescent males. Developmental Psychology, 5(9), 1216-1225. http://dx.doi.org/10.1037/dev0000027

Durá, T. y Castroviejo, A. (2011). Adherencia a la dieta mediterránea en la población universitaria. Nutrición Hospitalaria, 26(3), 602-608. http://dx.doi.org/10.3305/nh.2011.26.3.4891

Eisenberg, M. E. y Neumark-Steiner, D. (2013). Dieting and encouragement to diet by significant other: associations with disordered eating in Young adults. American Journal Health Promotion, 27(6), 370377. http://dx.doi.org/10.4278/ajhp.120120-QUAN57 
Encuesta Nacional de Ingesta Dietética (2014). En Observatorio de la Nutrición y de Estudio de la Obesidad (NAOS, 2014). Madrid, España: Ministerio de Sanidad, Servicios Sociales e Igualdad. Recuperado de: http:/www.aecosan.msssi.gob.es/

Goldschmidt, A. B., Wall, M., Loth, K. A., Le Grange, D. y Neumark-Sztainer, D. (2012). Wich dieters are at risk for the onset of binge eating? a prospective study of adolescents and young adults. Journal of Adolescent Health, 51(1), 86-92. Recuperado de http://www.researchgate.net/publication/228061171

Kortabarria, L., Maganto, C. y Peris M. (2013). Imagen corporal, autoestima y dietas en adolescentes. En J. J. Gázquez, C. Pérez, M. M. Molero y R. Parra (Eds.), Investigación en el ámbito escolar. Un acercamiento multidimensional a las variables psicológicas y educativas (pp. 707-712). Granada, España: Grupo Editorial Universitario.

Liechty, J. M. y Lee, M. (2013). Longitudinal predictors of dieting and disordered eating among young adults in the U.S. International Journal of Eating Disorders, 46(6), 790-800. http://dx.doi.org/10.1002/eat.22174

Loth, K. A., MacLehose, R., Bucchianeri, M., Crow, S. y Neumark-Sztainer, D. (2014). Predictors of dieting and disordered eating behaviors from adolescence to young adulthood. Journal of Adolescent Health, 55, 705-712. http://dx.doi.org/10.1016/j.jadohealth

Maganto, C. y Cruz, S. (2008). TSA Test de Siluetas para Adolescentes. Madrid, España: TEA.

Morales, P. (2011). Guía para construir cuestionarios y escalas de actitudes. Guatemala: Universidad Rafael Landívar. Recuperado de http://www.upcomillas.es/personal/peter/otrosdocu mentos/Guiaparaconstruirescalasdeactitudes.pdfíN DICE

Neumark-Sztainer, D., Wall, M., Haines, J., Story, M. y Eisenberg, M. E. (2007). Why does dieting predict weight gain in an adolescent? Findings from Project EAT-II: A 5-year longitudinal study. Journal of the American Dietetic Association. 107, 448-455. http://dx.doi.org/10.1016/j.jada.2006.12.013

Racine, S. E., Burt, S., Iacono, A., McGue, M. y Klump, K. (2011). Dietary restraint moderates genetic risk for binge eating. Journal of Abnormal Psychology, 120(1), 119-128. http://dx.doi.org/10.1037/a0020895

Rodríguez, F., Palma, X., Romo, A., Escobar, D., Aragú, B., Espinoza, L., ... y Gálvez, J. (2013). Hábitos alimentarios, actividad física $\mathrm{y}$ nivel socioeconómico en estudiantes universitarios de Chile. Nutrición Hospitalaria, 28(2), 447-455. http://dx.doi.org/10.3305/nh.2013.28.2.6230

Saucedo, T. y Unikel, C. (2010). Conductas alimentarias de riesgo, interiorización del ideal estético de delgadez e índice de masa corporal en estudiantes hidalguenses de preparatoria y licenciatura de una institución privada. Salud Mental, 33(1), 11-19.

Sehm, M. y Warschburger, P. (2015). The specificity of psychological factors associated with binge eating in adolescent boys and girls. Journal of Abnormal Child Psychology, 43, 1563-1571. http://dx.doi.org/10.1007/s10802-015-0026-7

Serra-Majem, L. L. y Aranceta, J. (2012). Alimentación infantil y juvenil. Barcelona, España: Toray Masson.

Timbo, A., Hormes, J. y Chubski, J. (2012). Will the real vegetarian please stand up? An investigation of dietary restraint and eating disorder symptoms in vegetarians versus non-vegetarians. Apetite, 58(3), 982-990.

http://dx.doi.org/10.1016/j.appet.2012.02.005 
